\title{
Perfil de idosos acometidos por câncer em cuidados paliativos em domicílio*
}

\author{
Elderly's profile affected by cancer in palliative care \\ at home \\ Perfil de mayores afectados por el cáncer en \\ cuidados paliativos en el hogar
}

Jossiana Wilke Faller Pedro Henrique Brusnicki Adriana Zilly Maria Célia Bezerra Ferrer Silva Brofman

Leila Cavalhieri

RESUMO: Objetivou-se identificar o perfil sociodemográfico e clínico de idosos com câncer em cuidados paliativos. Estudo descritivo, quantitativo, realizado em janeiro de 2015, em Foz do Iguaçu, PR, por meio de um instrumento estruturado. Dos 321 prontuários analisados, houve predominância de homens, acima dos 70 anos, de baixa escolaridade, com câncer de próstata, tratados com uso de paracetamol e codeína. Os dados subsidiam novas ações baseadas em critérios científicos e de qualidade a essa população.

Palavras-chave: Enfermagem; Cuidados Paliativos; Oncologia.

ABSTRACT: The aim of this work was to identify the sociodemographic profile of seniors in CP treated at home. Quantitative and descriptive research, that was done in January (2015), in Foz do Iguaçu, PR, using a structured instrument. From 321 medical records analyzed, was a predominance of men over 70 years, low education, with prostate cancer, treated, in use of paracetamol and codeine. It was concluded that this study might contribute to improving and creating new proposals for health action for this population.

Keywords: Nursing; Palliative Care; Medical Oncology.

* Com fomento da Universidade Estadual do Oeste do Paraná, PRPPG.

Faller, J. W., Brusnicki, P. H., Zilly, A., Brofman, M. C. B. F. S., \& Cavalhieri, L. (2016, janeiro). Perfil de idosos acometidos por câncer em cuidados paliativos no domicílio. Revista Kairós Gerontologia,19(N. ${ }^{\circ}$ Especial 22,

“Envelhecimento e Velhice”), pp. 29-43. ISSNe 2176-901X. São Paulo (SP), Brasil: FACHS/NEPE/PEPGG/PUC-SP 
RESUMEN: El objetivo de este trabajo fue identificar el perfil sociodemográfico y clínico de las personas mayores con cáncer en los cuidados paliativos. Este estudio descriptivo se realizó en enero de 2015 en Foz de Iguazú, PR, a través de un instrumento estructurado. De las 321 historias clínicas analizadas, hubo un predominio de los hombres mayores de 70 años, de baja escolaridad, con cáncer de próstata tratados con el uso de paracetamol y codeína. Los datos apoyan las nuevas medidas basadas en criterios científicos y calidad a esta población.

Palabras clave: Enfermería; Cuidados paliativos; Oncología médica.

\section{Introdução}

O envelhecimento é uma realidade atual na maioria das sociedades, em que se desenvolve como um fenômeno mundial. No Brasil, ocorre de forma acelerada, o que provocará mudanças nos serviços de saúde, na sociedade e na família. Estima-se, segundo o Instituto Brasileiro de Geografia e Estatística (IBGE), que existam 19 milhões de idosos, aproximadamente $10,8 \%$ da população (Barros, Maia, \& Pagliuca, 2011). Com o aumento da longevidade, ocasionado pela queda da fecundidade, da mortalidade, e da maior expectativa de vida, há o maior acometimento de doenças crônicas que, nos idosos, tendem a ser múltiplas, revelando sinal de alerta e de necessidade de preparo por parte dos profissionais, os quais passam a lidar com uma variedade de fatores de risco e incapacidades, elevando a demanda pelos serviços (Oliveira, Bozzetti, Hauser, Duncan, \& Harzheim, 2013).

O envelhecimento é um processo multifatorial e é, hoje, o maior fator de risco para o desenvolvimento do câncer. Indivíduos com 65 anos ou mais têm um aumento de 11 vezes na incidência de câncer, e 16 vezes na mortalidade, quando comparados com aqueles com idade inferior. Além disso, $70 \%$ das mortes por câncer no mundo já ocorrem em quem tem mais de 65 anos (Mohile, Nagovskiy, \& Balducci, 2009), o que revela sua magnitude epidemiológica, social e econômica na população e, em especial nos idosos, o que fomenta investigações e pesquisas para minimizar o impacto à saúde desta população. 
O câncer pode ter fatores intrínsecos, extrínsecos, ou uma combinação de ambos, o que leva a confirmar que a população idosa, por ter sofrido maior exposição a inúmeros fatores, está alguma forma mais susceptível ao câncer (MS, 2011). Essa susceptibilidade às doenças crônicas nesses indivíduos ocorre também por alterações fisiológicas, decorrentes do próprio processo de envelhecimento, e do declínio das funções orgânicas e, consequentemente, de sua qualidade de vida, o que pode, dessa forma, levá-los a um final de vida (Fratezi, \& Gutierrez, 2011).

Nesse contexto de final de vida diante de um prognóstico não favorável, inserem-se os Cuidados Paliativos (CP) como importante e nova modalidade de cuidar. Os CP são medidas não curativas, aplicadas em pacientes cujo avanço da enfermidade provoca sinais e sintomas debilitantes e causadores de sofrimento, tanto para o paciente quanto para os cuidadores/familiares.

Profissionais das ciências da saúde, sociais, e humanas atuam de forma multiprofissional, atuando em âmbito domiciliar e hospitalar, com intervenções na saúde global. Como o paliativismo não visa à cura pode ser aplicado independentemente do prognóstico da enfermidade, inclusive, em conjunto com o tratamento curativo, pois a prioridade é oferecer a melhor qualidade de vida possível ao doente e sua família (Fonseca, A.C., \& Fonseca, M.J.M., 2010), alívio do sofrimento, da identificação precoce dos cuidados, da avaliação impecável e tratamento da dor e demais sintomas físicos, sociais, psicológicos e espirituais (WHO, 2002).

Os pacientes em final de vida, habitualmente, apresentam múltiplos sintomas, por vezes intensos e debilitantes e se torna fundamental a avaliação, de forma a serem instituídas intervenções adequadas que visem ao seu controle (Souza, 2012). A Associação Europeia de Cuidados Paliativos (2010) complementa, referindo estes cuidados como sendo ativos, totais, e com uma abordagem interdisciplinar, incluindo o doente, a família e a comunidade, com o propósito de satisfazer as necessidades do paciente.

Visto que o crescimento da população idosa no país aumentou, e com isso, o número de idosos com neoplasias e sua frequente taxa de mortalidade por essa causa, o objetivo deste estudo foi identificar o perfil sociodemográfico e clínico de idosos com câncer em cuidados paliativos, em um serviço público de atendimento paliativo oncológico no município de Foz do Iguaçu, PR, e apresentar uma elaboração reflexiva sobre a questão. 


\section{Metodologia (materiais e métodos)}

Trata-se de um estudo transversal, descritivo e de abordagem quantitativa, desenvolvido em um serviço de atendimento a pacientes oncológicos em cuidados paliativos, atendendo pacientes de um Centro Especializado de Oncologia, no município de Foz do Iguaçu, PR, referência no acompanhamento de pacientes com neoplasias malignas para os nove municípios circunvizinhos que integram a 9a Regional de Saúde do Paraná.

O Grupo Interdisciplinar de Suporte Oncológico, GISO, criado em 2001, tem como público-alvo os pacientes da oncologia, com necessidade de cuidados paliativos, em geral, egressos hospitalares, que recorrem com frequências às portas de urgência.

O programa realiza a assistência, por meio de visitas domiciliares, com visitas periódicas, por encaminhamento médico ou solicitação do paciente e/ou cuidador.

A equipe conta com profissionais da área médica, da enfermagem e da psicologia, uma equipe multidisciplinar e necessariamente bem-integrada.

O objetivo é melhorar a qualidade de vida do paciente em fase terminal, além de instrumentalizar o cuidador, reduzindo a necessidade de internação. Essa assistência inclui consulta médica e de enfermagem, realização de hidratação venosa, curativos, cuidados com sondas e cateteres, além do atendimento psicológico, de modo que alcance as necessidades de cada indivíduo.

O programa realiza um intercâmbio com o programa Estratégia de Saúde da Família, ESF e Centro de Referência de Assistência Social, CRAS, no intuito de prover a assistência aos pacientes, oferecendo aporte de cuidados e auxílio com materiais, consultas, fármacos, entre outros.

O CRAS oferece orientação e auxílio relacionados à aposentadoria entre outros serviços sociais. O número de pacientes cadastrados em tratamento/acompanhamento até janeiro de 2015 era de 717 usuários.

Foz do Iguaçu é uma região de tríplice fronteira, vizinho dos países Paraguai e Argentina e que possuía, no ano de 2010, aproximadamente 256.088 habitantes e 18.447 idosos (7,2\%) (IBGE, 2010). Possui 28 unidades de saúde, 19 unidades de saúde da família, cinco centros de referência, CRF, e quatro unidades básicas de saúde. 
Os dados do estudo foram coletados no mês de janeiro de 2015, por meio de um instrumento estruturado, com dados sociodemográficos (sexo, idade, estado civil, escolaridade, ocupação) e clínicos (diagnóstico, tempo de diagnóstico, tratamento utilizado e uso de fármacos), retirados dos prontuários.

Os critérios adotados para inclusão no estudo foram pacientes com idade igual ou superior a 60 anos, residentes no município, uma vez que o programa atende indivíduos de municípios vizinhos e com cadastros ativos até a data da coleta.

Para a análise dos dados, estes foram transcritos para uma planilha no Microsoft Word Excel ${ }^{\circledR}$, codificadas e analisadas por estatística descritiva.

A pesquisa ocorreu em conformidade com o preconizado pela Resolução 466/12 do Conselho Nacional de Saúde e contou com a aprovação do Comitê de Ética em Pesquisa em Seres Humanos (CONEP) da Universidade Estadual do Oeste do Paraná, UNIOESTE, sob parecer n. ${ }^{\circ} 861.927 / 2014$.

\section{Resultados}

Foram identificados 321 idosos, correspondendo 44,8\% dos pacientes atendidos pelo programa. A maioria encontra-se na faixa etária dos 70 a 79 anos (n=142/44,2\%), com predominância do sexo masculino $(n=168 / 52,3 \%)$, com ensino fundamental incompleto $(n=187 / 58,3 \%)$, casados $(n=191 / 59,5 \%)$, em sua maioria do lar $(\mathrm{n}=87 / 27,1 \%)$, e aposentados $(\mathrm{n}=85 / 26,5 \%)$, naturais dos estados do Sul do Brasil $(n=215 / 67 \%)$ e $3,7 \%(n=12)$ e provenientes de outros países, como Paraguai, Argentina, Líbano e Portugal, já que o município encontra-se em região de fronteira, agregando cerca de 79 diferentes nacionalidades (BRASIL, 2008). Apenas 27,1\% eram tabagistas $(\mathrm{n}=87)$, e $17,5 \%$ etilistas $(\mathrm{n}=56)$.

Na distribuição dos idosos por distritos do município, observa-se um número significativo de pacientes residentes na região leste $(n=112 / 34,9 \%)$, os quais podem ser analisados na tabela 1. 
Tabela 1. Perfil sociodemográfico de idosos em cuidados paliativos. Foz do Iguaçu, PR, 2015

\begin{tabular}{|c|c|c|c|c|c|}
\hline Faixa etária & $\mathbf{N}$ & $\%$ & Tabagismo & $\mathbf{N}$ & $\%$ \\
\hline $60-69$ & 123 & 38,3 & Sim & 087 & 27,1 \\
\hline $70-79$ & 142 & 44,2 & Não & 075 & 23,4 \\
\hline 80 e mais & 056 & 17,5 & Ignorado & 159 & 49,5 \\
\hline Sexo & & & Etilismo & & \\
\hline$M$ & 168 & 52,3 & Sim & 056 & 17,5 \\
\hline \multirow[t]{2}{*}{$\mathrm{F}$} & 153 & 47,7 & Não & 103 & 032 \\
\hline & & & Ignorado & 162 & 50,5 \\
\hline Estado Civil & & & Escolaridade & & \\
\hline Ignorado & 008 & 02,5 & Analfabeto & 061 & 019 \\
\hline Solteiro (a) & 028 & 08,7 & EFI/ EFC & 205 & 63,9 \\
\hline Casado (a) & 192 & 59,8 & EMI/ EMC & 022 & 06,8 \\
\hline Viúvo (a) & 069 & 21,5 & ESI/ ESC & 008 & 02,5 \\
\hline Divorciado (a) & 024 & 07,5 & Ignorado & 025 & 07,8 \\
\hline Distritos & & & Naturalidade & & \\
\hline Leste & 112 & 34,9 & Ignorado & 002 & 00,6 \\
\hline Norte & 082 & 25,5 & Sul & 215 & 067 \\
\hline Sul & 047 & 14,6 & Sudeste & 063 & 19,6 \\
\hline Oeste & 040 & 12,5 & Nordeste & 023 & 07,1 \\
\hline \multirow[t]{2}{*}{ Nordeste } & 040 & 12,5 & Centro-Oeste & 006 & 01,9 \\
\hline & & & Outros países & 012 & 03,7 \\
\hline Profissão & & & Tempo de diagn & & \\
\hline Do Lar & 087 & 27,1 & $\leq 4$ anos & 115 & 35,8 \\
\hline Aposentado & 085 & 26,5 & $>4 \leq 9$ anos & 168 & 52,4 \\
\hline Outros & 137 & 42,7 & $>9$ anos & 037 & 11,5 \\
\hline Ignorado & 012 & 03,7 & Ignorado & 001 & 00,3 \\
\hline $\begin{array}{l}\text { Localização } \\
\text { Sexo Feminino }\end{array}$ & & & Tratamento & & \\
\hline Mama & 053 & 16,5 & Quimioterapia & 041 & 12,8 \\
\hline Colo Uterino & 028 & 08,7 & Radioterapia & 024 & 07,5 \\
\hline Estômago & 011 & 03,5 & Cirurgia & 033 & 10,3 \\
\hline Reto & 011 & 03,5 & Associação & 116 & 36,1 \\
\hline Demais & 043 & 13,4 & Ignorado & 107 & 33,3 \\
\hline \multicolumn{6}{|l|}{ Sexo Masculino } \\
\hline Próstata & 046 & 14,3 & & & \\
\hline Pulmão & 028 & 08,7 & & & \\
\hline Esôfago & 021 & 06,5 & & & \\
\hline Pele/Melanoma & 012 & 03,7 & & & \\
\hline Demais & 068 & 21,2 & & & \\
\hline Total & 321 & 100 & & 321 & 100 \\
\hline
\end{tabular}

Nota: EFI: Ensino Fundamental Incompleto; EFC: Ensino Fundamental Completo; EMI: Ensino Médio Incompleto; EMC: Ensino Médio Completo; ESI: Ensino Superior Incompleto; ESC: Ensino Superior Completo. 
Em relação aos dados clínicos, as neoplasias de maior recorrência no sexo feminino foram a de mama $(n=51 / 15,9 \%)$ e colo uterino $(n=28 / 8,7 \%)$; e, no sexo masculino, verificamos próstata $(n=46 / 14,3 \%)$, seguido de pulmão ( $n=20 / 6,2 \%$ ) e esôfago ( $\mathrm{n}=15 / 4,6 \%)$. As demais neoplasias incluíram Pele/Melanoma, Estômago, Reto, Laringe, Cólon, Língua, Linfoma Não Hodgking, Mieloma Múltiplo, Endométrio, Nasofaringe, Amigdala, Boca, Coluna Vertebral, Pâncreas, Sarcoma, Ovário, Cérebro, Hipofaringe, Orofaringe, Seio Piriforme, Leucemia/Tricoleucemia, Rim, Tecido Conjuntivo, Doença de Hodgking, Epiderme da Face, Gânglios Linfáticos, Gengiva, Orelha, Palato, Retrocricoide, Bexiga, Exocérvix, Fígado, Pênis, Perianal, Vagina, Vesícula Biliar e Tireoide, em ordem de maior prevalência de acometimento.

A estratégia terapêutica de maior escolha para os casos em estudo foi a associação, a qual reúne a quimioterapia e a radioterapia, aliados ou não à cirurgia $(n=116 / 36,1 \%)$, os quais estão apresentados na tabela 1 .

Os fármacos mais utilizados foram os opioides e suas associações (Paracetamol/Codeína) n=226/70,4\%, antiácidos e inibidores da secreção gástrica ( $n=244 / 76 \%)$, antieméticos e agentes procinéticos $(n=240 / 74,7 \%)$, antiinflamatórios (esteroides e não esteroides) (n=67/20,8\%), antidepressivos $(n=63 / 19,6 \%)$ e antiespasmódicos $(n=53 / 16,5 \%)$. Dentre o total de pacientes do estudo, 28 (8,7\%) não faziam uso de fármacos ou não constava a prescrição médica em sua ficha cadastral (Quadro 1).

Quadro 1. Fármacos utilizados em idosos em cuidados paliativos. Foz do Iguaçu, PR, 2015

\begin{tabular}{|c|c|c|c|}
\hline Classe do Fármaco & Fármacos mais utilizados & $\mathbf{N}$ & $\mathbf{\%}$ \\
\hline $\begin{array}{c}\text { Antiácidos e Inibidores de } \\
\text { Secreção Gástrica }\end{array}$ & Omeprazol/Ranitidina & 238 & 74,1 \\
\hline $\begin{array}{c}\text { Analgésicos (Opióides e } \\
\text { associações) }\end{array}$ & Paracetamol/Codeína & 226 & 70,4 \\
\cline { 2 - 4 } & Dipirona/Morfina & 094 & 29,3 \\
\hline $\begin{array}{c}\text { Antieméticos e Agentes } \\
\text { Procinéticos }\end{array}$ & Metoclopramida/Digesan & 197 & 61,4 \\
\hline $\begin{array}{c}\text { Antidepressivos } \\
\text { Antiespasmódicos }\end{array}$ & Amitriptilina & 062 & 19,3 \\
\hline AINE/AIE & Dexametazona/Prednizona & 054 & 16,5 \\
\hline Laxantes e Antiflatulentos & Óleo Mineral/Luftal/Florax & 019 & 05,9 \\
\hline
\end{tabular}

Nota: AINE: Anti-Inflamatórios Não Esteroides; AIE: Anti-Inflamatórios Esteroides 


\section{Discussão}

O processo de envelhecimento repercute em inúmeras modificações que acontecem na saúde, em especial as doenças crônicas não-transmissíveis, como o câncer (Visentin, \& Lenardt, 2010). Um estudo realizado por Vianna, Silva Marconato, Gindri, Beneton, Lima, \& Viero (2013) revelou um aumento da incidência de casos de câncer na população idosa, sendo o câncer de mama na população feminina $(30,13 \%)$; e o de próstata $(33,33 \%)$, na população masculina.

Esses dados coincidem com as estatísticas atuais pesquisadas para o Brasil e para o mundo (INCA, 2011), em que 70\% dos diagnósticos de câncer no Brasil ocorrem em indivíduos acima de 60 anos, sendo que aproximadamente $60 \%$ tem mais de 70 anos, o que corrobora também com o estudo em relação à faixa etária e as neoplasias prevalentes.

O câncer de mama é o mais acometido em mulheres, totalizando uma estimativa de 10.370 novos casos. A neoplasia de pulmão segue em segundo lugar na estimativa para os homens com 4.720 novos casos e, em terceiro, para as mulheres com 3.110 (INCA, 2014). De acordo com o DATASUS, cujos dados são de 2011, 153 das mortes por neoplasia em homens no Paraná, na faixa etária entre 60-69 anos, ocorreu devido ao câncer de próstata; 332 na faixa etária entre 70-79 anos e 376 a partir de 80 anos. Para as mulheres, 59 mortes por câncer de colo do útero entre 60-69 anos, 54 entre 70-79 anos e 17 a partir dos 80 anos (Brasil, 2012). No município de Foz do Iguaçu (PR), os idosos em CP revelam a mesma incidência nacional, o que sustenta a elaboração e implementação de políticas públicas para prevenção e promoção da saúde da população adulta jovem, no sentido de prevenir ou retardar agravos.

Os dados deste estudo mostraram que os idosos apresentam o diagnóstico da doença por 4 a 9 anos, e o maior período foi de 17 anos de diagnóstico. Pode-se afirmar que, ainda que o câncer seja um dos principais causadores de morte entre os idosos, isso não significa que o período entre diagnóstico e o óbito seja breve, reforçando a importância de uma abordagem multiprofissional, considerando as comorbidades associadas e o cuidado integral.

Relativamente à predominância do sexo masculino, as estimativas do INCA para o ano de 2014, na região Sul do Brasil, eram de 66.540 novos casos de neoplasia entre homens, comparado a 49.790 casos entre as mulheres (INCA, 2014). 
De acordo com alguns estudos relacionados ao diagnóstico de câncer, a baixa escolaridade, associada à baixa renda, são fatores que interferem no acesso aos serviços de saúde, causando a demora da procura pela assistência à saúde, e diminuindo o número de exames preventivos como mamografia, exame de Papanicolau e da próstata (Paiva, Motta, \& Griep, 2010; Höfelmann, Anjos, \& Ayala, 2014; Thuler, Bergmann, \& Casado, 2012). Os dados do estudo confirmam essa panorâmica, ao revelar os homens como mais prevalentes e de baixa escolaridade.

Em relação ao tabaco, em uma revisão de mais de 150 artigos no ano de 2009, vários tumores foram confirmados como associados a essa exposição, além de evidências que demonstram um risco aumentado para outros tantos (Zelmanowicz, Moreira, \& Silva, 2012). O alcoolismo tem sido associado ao câncer de esôfago, laringe e faringe, mas também pode ser um fator de risco para o câncer de próstata. Embora o etanol não seja um cancerígeno direto, um dos seus metabólitos, o acetaldeído, pode atuar como um propulsor tumoral. Além disso, o uso abusivo do álcool coopera com o aparecimento de hepatite B crônica ou infecção com o vírus da hepatite $\mathrm{C}$, predispondo, assim, o organismo para o desenvolvimento do carcinoma hepatocelular (Paz, et al., 2014).

Ainda em relação aos dados sociodemográficos, nota-se que a maior parte da população estudada é residente do distrito leste do município. Analisando o mapa de estabelecimentos de saúde, é possível identificar que, nesse distrito, há 5 unidades básicas de saúde, 1 centro de referência da família, 1 pronto-atendimento, 1 centro de atenção psicossocial II (CAPS II) e 1 ambulatório de saúde mental, totalizando 9 locais de assistência ao usuário. Nos demais distritos, há um número menor de estabelecimentos de saúde, entre 4 a 8 unidades. Esse fato sugere a ocorrência de mais diagnósticos nessa área pela maior acessibilidade aos serviços de saúde e equipe de saúde mais atuante.

Além da oferta de serviços, essencial na Atenção Primária em Saúde (APS), o perfil socioeconômico dessa população é de trabalhadores do comércio e da economia informal, da construção civil e de prestação de serviço do setor terciário. Possui cerca de 51 bairros e o maior agregado populacional do município, com cerca de 93.000 habitantes (Foz do Iguaçu, 2010).

Em relação às opções terapêuticas reveladas no estudo, houve predominância do uso associativo da quimioterapia, radioterapia e cirurgia. 
O Instituto Nacional do Câncer (INCA, 2015) relata que a opção terapêutica para o câncer de mama depende do estadiamento da doença, inclusive fatores como idade, tamanho tumoral e grau de diferenciação celular. Se o diagnóstico foi realizado na fase inicial da doença, tratamentos localizados como cirurgia e radioterapia podem apresentar maior eficácia. Contudo, quando a doença se apresenta em estadio avançado ou metastático, os tratamentos sistêmicos como quimioterapia e hormonioterapia são recomendados (INCA, 2015).

Portanto, isso leva a inferir que os idosos do estudo, em sua maioria, apresentaram diagnóstico tardio, o que levou a necessidade de tratamentos sistêmicos. Esse método também se aplica no tratamento do câncer de próstata. Além dos tratamentos sintomáticos oferecidos durante os $\mathrm{CP}$, a cirurgia, a radioterapia, a hormonioterapia e a quimioterapia podem ser utilizadas com finalidade paliativa (Souza, 2011).

Para controle dos efeitos adversos da terapêutica, como dor, náusea, diarreia, inapetência, entre outros, os pacientes utilizam uma gama de fármacos para controle dos sintomas, como inibidores da secreção gástrica, analgésicos opióides, muito associados aos analgésicos não opióides, para controle da dor, antieméticos devido a náuseas e êmese, antidepressivos, entre outros. O uso desses medicamentos é um ponto importante dentro dos $\mathrm{CP}$, pois ao se diminuírem os sintomas adversos do tratamento, aumenta-se a qualidade de vida do paciente, ainda que não seja possível a cura.

Nos cuidados paliativos, a morte é compreendida como parte natural da vida humana e esperada quando ocorre uma doença sem cura. Mostra-se como possibilidade de preencher as lacunas nos cuidados ativos às pessoas nessas condições (Matsumoto, 2012). Nesse sentido, os cuidados paliativos consistem em fornecer atenção diferenciada aos doentes e seus familiares, diante de patologias que revelam a possibilidade de morte, seja em curto ou longo prazo, objetivando também proporcionar finitude digna, com qualidade para o tempo de convivência entre a pessoa e uma doença sem cura, e suas repercussões nas esferas física, psíquica, emocional, social, ocupacional e espiritual (Souza, 2014). Os CP possuem uma filosofia que pode ser aplicada em diferentes contextos institucionais, ou seja, pode ser realizada tanto nas instituições de saúde quanto no domicílio (Andrade, et al., 2012). 
A função do GISO é levar os CP até o local onde o paciente está inserido em âmbito familiar, oferecendo assistência interdisciplinar e, assim, diminuindo a frequência com que esses pacientes recorrem ao serviço de saúde de urgência e emergência. Contudo, a internação pode se fazer necessária, levando em consideração a complexidade dos processos terapêuticos voltados a essa enfermidade (Rocha, 2011).

Outro fator que leva à internação recorrente é a falta de preparo dos cuidadores/familiares para oferecer esse cuidado, causando sobrecarga e angústia aos familiares (Ali, 2011).

Isso só afirma a necessidade de uma atenção especializada e disposta a orientar e dar suporte a essas famílias; assim, os pacientes podem permanecer em um ambiente de menores riscos, em contato com a família e recebendo assistência que ofereça uma qualidade de vida.

O Programa Nacional de Cuidados Paliativos de Portugal (2010) afirma que os CP têm como ferramentas essenciais o alívio dos sintomas; o apoio psicológico, espiritual e emocional; o apoio à família; o apoio durante o luto; e a interdisciplinaridade. Barbosa e Neto (2010) mencionaram quatro áreas fundamentais para a aplicação de cuidados paliativos, sendo (1) o controle de sintomas; (2) z comunicação adequada; (3) o apoio à família; e (4) o trabalho em equipe. Estas quatro áreas são fundamentais, não sendo possível a prática de cuidados paliativos de qualidade se alguma delas for depreciada.

Desse modo, após análise de dados secundários desses indivíduos, observa-se que, diante de um deficit da estrutura e de poucos profissionais atuantes no programa, é possível oferecer os princípios preconizados para a assistência humanizada e levar benefícios aos pacientes e seus familiares, diante de uma doença complexa que ameaça a continuidade da vida. Não há dúvidas que CP é uma necessidade de saúde pública e humanitária.

\section{Conclusão}

Este estudo possibilitou a caracterização do perfil sociodemográficos de idosos em CP atendidos em âmbito domiciliar no município de Foz do Iguaçu, PR. 
Os resultados apontaram que essa população apresenta, em sua maioria, uma faixa etária avançada, com baixa escolaridade, um longo período de tratamento da doença e utilizam uma grande quantidade de fármacos para diminuir a dor e outros sintomas relacionados ao câncer. Houve prevalência do sexo masculino, e as neoplasias de maior incidência foram de mama no sexo feminino; e próstata para o sexo masculino.

Os $\mathrm{CP}$, promovidos no domicílio, permitem ao idoso a possibilidade de continuar no seu contexto social e familiar, recebendo atenção interdisciplinar, evitando assim as internações recorrentes e melhorando sua qualidade de vida.

Além disso, essa modalidade de cuidado também é favorável para o atendimento institucional, pois descongestiona as salas de pronto-atendimento ambulatoriais e hospitalares. As vantagens de uma assistência paliativista a esses pacientes e familiares permite orientação e apoio aos envolvidos para no período final de vida. Os $\mathrm{CP}$ não reduzem nem aumentam o tempo de vida desses idosos, mas permitem que vivam com menos dor e sofrimento até o momento do óbito.

Portanto, analisar o perfil desses idosos subsidia melhoria da atenção prestada, de modo a embasar novas propostas de ação que solidifiquem o serviço no município, para prover ainda mais a qualidade de vida a essa população.

\section{Referências}

Ali, A. M. A. S. A. (2011). Cuidados Paliativos e a Saúde dos Idosos no Brasil. São Paulo, SP: PUC-SP: Revista Kairós Gerontologia, 14(1), 125-136. Recuperado em 01 maio, 2015, de: http://revistas.pucsp.br/index.php/kairos/article/viewFile/6931/5023.

Andrade, C. G., Santos, K. F. O., Costa, S. F. G., Fernandes, M. D. G. M., Lopes, M. E. L., \& Souto, M. C. (2012). Cuidados Paliativos ao Paciente Idoso: uma Revisão Integrativa da Literatura. Revista Brasileira de Ciências da Saúde, 16(3), 411-418. Recuperado em 01 maio, 2015, de: http://periodicos.ufpb.br/index.php/rbcs/article/viewFile/12587/7883.

Barbosa, A., \& Neto, I. G. (2010). Manual de Cuidados Paliativos. (2 ${ }^{\mathrm{a}}$ ed.). Lisboa, Portugal: Faculdade de Medicina da Universidade de Lisboa.

Barros, T. B., Maia, E. R., \& Pagliuca, L. M. F. (2011). Facilidades e dificuldades na assistência ao idoso na estratégia de saúde da família. Rev. Rene, Fortaleza, 12(4), 732-741. Recuperado em 01 de maio, 2015, de: http://www.revistarene.ufc.br/vol12n4_pdf/a10v12n4.pdf.

Brasil. (2008). Departamento de Polícia Federal. Sistema Nacional de Cadastramento de Registro de Estrangeiros (SINCRE). Brasília, DF.

(2011). Ministério da Saúde. Instituto Nacional de Câncer (Brasil). ABC do câncer: abordagens básicas para o controle do câncer. Rio de Janeiro: INCA, 128 p.

Faller, J. W., Brusnicki, P. H., Zilly, A., Brofman, M. C. B. F. S., \& Cavalhieri, L. (2016, janeiro). Perfil de idosos acometidos por câncer em cuidados paliativos no domicílio. Revista Kairós Gerontologia,19(N. ${ }^{\circ}$ Especial 22,

“Envelhecimento e Velhice”), pp. 29-43. ISSNe 2176-901X. São Paulo (SP), Brasil: FACHS/NEPE/PEPGG/PUC-SP 
(2012). Ministério da Saúde. Indicadores de mortalidade. Taxa de mortalidade específica por neoplasias malignas. Recuperado em: 30 maio, 2015, de: http://bit.ly/1K2UpAJ.

(2014). Ministério da Saúde. Instituto Nacional de Câncer (Brasil). Estimativa 2014: Incidência de Câncer no Brasil. Instituto Nacional de Câncer José Alencar Gomes da Silva, Coordenação de Prevenção e Vigilância. Rio de Janeiro.

(2015). Ministério da Saúde. Instituto Nacional de Câncer (Brasil). Tratamento do Câncer. Recuperado em 18 junho, 2015, de: http://goo.gl/2mwPT2.

European Association for Palliative Care. (2010). Recuperado em 06 junho, 2015, de: http://www.eapcnet.eu.

Fonseca, A. C., \& Fonseca, M. J. M. (2010). Cuidados paliativos para idosos na unidade de terapia intensiva: realidade factível. Porto Alegre, RS: Scientia Medica, 20(4), 301309. Recuperado em 01 maio, 2015, de: http://www.scielo.br/pdf/csc/v16n7/23.pdf.

Foz do Iguaçu (2010). Prefeitura do município de Foz do Iguaçu, PR. Secretaria Municipal da Saúde. Plano Municipal de Saúde 2010-2013. Recuperado em 01 maio, 2015, de: http://www.pmfi.pr.gov.br/Portal/VisualizaObj.aspx?IDObj=10950.

Fratezi, F. R., \& Gutierrez, B. A. O. (2011). Cuidador familiar do idoso em cuidados paliativos: o processo de morrer no domicílio. Ciênc. Saúde Colet., 16(7), 3241-3248. Recuperado em 01 maio, 2015, de: http://www.scielo.br/pdf/csc/v16n7/23.pdf.

Höfelmann, D. A., Anjos, J. C., \& Ayala, A. L. (2014). Survival for ten years and prognostic factors for women with breast cancer in Joinville in the State of Santa Catarina, Brazil. Ciência \& Saúde Coletiva, 19(6), 1813-1824. Recuperado em 01 maio, 2015, de: http://www.scielosp.org/pdf/csc/v19n6/1413-8123-csc-19-06-01813.pdf.

INCA. (2011). Instituto Nacional de Câncer José Alencar Gomes da Silva. Recuperado em 01 maio, 2015, de:

http://www2.inca.gov.br/wps/wcm/connect/agencianoticias/site/home/noticias/2011/inc a_lanca_estimativas_de_cancer_2012_com_sete_novas_localizacoes_de_tumores.

INCA. (2014). Instituto Nacional de Câncer José Alencar Gomes da Silva. Recuperado em 01 maio, 2015, de: http://www.inca.gov.br/bvscontrolecancer/publicacoes/Estimativa_2014.pdf.

INCA. (2015). Instituto Nacional de Câncer José Alencar Gomes da Silva. Recuperado em 01 maio, 2015, de: http://www.inca.gov.br/wcm/outubro-rosa/2015/cancer-demama.asp.

IBGE. (2013). Instituto Brasileiro de Geografia e Estatística. Pesquisa Nacional de Saúde 2013: Percepção do estado de saúde, estilos de vida e doenças crônicas. Recuperado em 20 maio, 2015, de: http://goo.gl/45Jxsp.

Matsumoto, D. Y. (2012). Cuidados Paliativos: conceitos, fundamentos e princípios. In: Carvalho, R. T., \& Parsons, H. A. (Orgs.). Manual de Cuidados Paliativos ANCP. (Ampl. Atual. $2^{\text {a }}$ ed.). São Paulo, SP: Academia Nacional de Cuidados Paliativos.

Mohile, S., Nagovskiy, N., \& Balducci, L. (2009). Chemotherapy for the Older Adult with Câncer. In: Hurria, A., \& Balducci, L. Geriatric Oncology: Treatment, Assessment and Management. Springer. 
Oliveira, E. B., Bozzetti, M. C., Hauser, L., Duncan, B. B., \& Harzheim, E. (2013). Avaliação da qualidade do cuidado a idosos nos serviços da rede pública de atenção primária à saúde de Porto Alegre, Brasil. Rio de Janeiro, RJ: Rev. Bras. Med. Fam. Comunidade, 8(29), 264-273. Recuperado em 01 maio, 2015, de: https://www.rbmfc.org.br/rbmfc/article/viewFile/826/586.

Paiva, E. P., Motta, M. C. S., \& Griep, R. H. (2010). Conhecimentos, atitudes e práticas acerca da detecção do câncer de próstata. Acta Paul. Enferm., 23(1), 88-93. Recuperado em 01 maio, 2015, de: http://www.scielo.br/pdf/ape/v23n1/14.pdf.

Paz, M. F. C. J., Monte, B. S., Neto, J. D. J. R., Tapety, F. I., Sousa, C. M. M., \& Cavalcante, A. A. D. C. M. (2014). Correlations between risk factors for prostate cancer: an epidemiological analysis. Revista de Pesquisa: Cuidado é Fundamental Online, 5(6), 187-199. Recuperado em 01 maio, 2015, de: http://www.seer.unirio.br/index.php/cuidadofundamental/.

Portugal. (2010). Ministério da Saúde, Programa Nacional de Cuidados Paliativos. Recuperado em 06 junho, 2015, de: http://goo.gl/5zNm3A.

Rocha, L. S. (2011). Idosos convivendo com câncer: possibilidades para o cuidado de si. (104 f.). Dissertação de mestrado em Enfermagem. Universidade Federal de Santa Maria, Centro de Ciências da Saúde, Rio Grande do Sul.

Souza, A. D. A. (2012). Sintomas em Cuidados Paliativos: Da Avaliação ao Controlo. (151f.). Dissertação de mestrado em Oncologia. Universidade do Porto, Instituto de Ciências Biomédicas Abel Salazar, Portugal.

Souza, G. G. A. (2014). Idosos hospitalizados e em cuidados paliativos oncológicos: possibilidades de fazer, ser e tornar-se, na finitude. (108 f.). Dissertação de mestrado em Psicologia. Instituto de Filosofia e Ciências Humanas, Universidade Federal do Pará, Belém, PA.

Souza, R. S. (2011). Pacientes Oncológicos em Quimioterapia Paliativa: perfil e relações entre sintomas, capacidade funcional e qualidade de vida. (96f.). Dissertação de mestrado em Enfermagem. Escola de Enfermagem, Universidade Federal de Minas Gerais, Belo Horizonte, MG.

Thuler, L. C. S., Bergmann, A., \& Casado, L. (2012). Perfil das pacientes com câncer do colo do útero no Brasil, 2000-2009: estudo de base secundária. Rev. Bras. Cancerol, 58(3), 351-357. Recuperado em 01 maio, 2015, de: http://www1.inca.gov.br/rbc/n_58/v03/pdf/04_artigo_perfil_pacientes_cancer_colo_uter o_brasil_2000_2009_estudo_base_secundaria.pdf.

Vianna, A. E., Silva Marconato, C., Gindri, L., Beneton, M. R., Lima, S. B. S., \& Viero, V. (2013). A enfermagem oncológica frente ao câncer em pacientes idosos. Revista Contexto \& Saúde, 11(20), 569-572. Recuperado em 01 maio, 2015, de: https://www.revistas.unijui.edu.br/index.php/contextoesaude/article/view/1580.

Visentin, A., \& Lenardt, M. H. (2010). O itinerário terapêutico: história oral de idosos com câncer. Acta paul. Enferm., 23(4), 486-492. Recuperado em 01 maio, 2015, de: http://www.scielo.br/pdf/ape/v23n4/07.pdf.

WHO. (2015). World Health Organization. Who Definition of Palliative Care. Recuperado em 15 abril, 2015, de: http://www.who.int/cancer/palliative/definition/en. 
Zelmanowicz, A. M., Moreira, J. S., \& Silva, L. C. C. (2012). Impacto do tabagismo no câncer. In: Da Silva, L. C. C. (Org.). Tabagismo: Doença que tem tratamento, 123-125. Porto Alegre, RS: Artmed.

Recebido em 05/07/2015

Aceito em 30/12/2015

Jossiana Wilke Faller - Mestre em Enfermagem pela Universidade Estadual de Maringá, PR. Docente na Unioeste, Universidade Estadual do Oeste do Paraná.

E-mail: jofaller@hotmail.com

Pedro Henrique Brusnicki - Graduando em Enfermagem na Unioeste, Universidade Estadual do Oeste do Paraná.

E-mail: pbrusnicki@ hotmail.com

Adriana Zilly - Doutora em Ciências. Docente nos Programa de Pós-Graduação Stricto Sensu de Saúde Pública em região de fronteira e Ensino da Unioeste, Universidade Estadual do Oeste do Paraná.

E-mail: aazilly@hotmail.com

Maria Célia Bezerra Ferrer Silva Brofman - Médica. Especialista em Cuidados Paliativos. Secretaria Municipal de Saúde. Responsável pelo Programa de Assistência Domiciliar e Grupo Interdisciplinar de Suporte Oncológico de Foz do Iguaçu, PR.

E-mail: celiabrofman@brturbo.com

Leila Cavalhieri - Enfermeira do Programa de Assistência Domiciliar e Grupo Interdisciplinar de Suporte Oncológico. Secretaria de Saúde do município de Foz do Iguaçu, PR.

E-mail: leilacava@hotmail.com 